Théologiques

Théologiques

\title{
La trame augustinienne dans Qu'est-ce que la religion? de Keiji Nishitani
}

\section{Vincent Giraud}

Volume 20, numéro 1-2, 2012

Les philosophes de l’École de Kyōto et la théologie

URI : https://id.erudit.org/iderudit/1018861ar

DOI : https://doi.org/10.7202/1018861ar

Aller au sommaire du numéro

\section{Éditeur(s)}

Faculté de théologie et de sciences des religions, Université de Montréal

\section{ISSN}

1188-7109 (imprimé)

1492-1413 (numérique)

Découvrir la revue

\section{Citer cet article}

Giraud, V. (2012). La trame augustinienne dans Qu'est-ce que la religion? de Keiji Nishitani. Théologiques, 20(1-2), 271-296.

https://doi.org/10.7202/1018861ar
Résumé de l'article

Nishitani Keiji (1900-1990) a mis en oeuvre une reprise féconde des questions propres à la tradition métaphysique occidentale en prenant appui sur la conception bouddhiste de la vacuité (japonais : $k \bar{u}$; sanscrit : śünyatā). Marchant sur les traces d'Augustin et de sa pensée de la conversion, il a lié aussi étroitement que possible pensée philosophique et quête religieuse. Le nouvel édifice conceptuel élaboré par Nishitani autour de la notion de śūnyatā ne prend en effet tout son sens que pour qui se rend capable d'une conversion au sens religieux du terme, décentrement radical de sa propre existence en direction de la vacuité, et permettant seul un accès véritable à l'être. La saisie par Nishitani de l'essence de la religion le conduit ainsi dans les parages de l'augustinisme, éclairant d'un jour nouveau le possible usage contemporain des catégories bouddhistes. 
Théologiques 20 (2012) p. 271-296

\title{
La trame augustinienne dans Qu'est-ce que la religion? de Keiji Nishitani
}

\author{
Vincent GIRAUD" \\ Philosophie de la religion \\ Université de Kyōto (Japon)
}

Qu'est-ce qu'un penseur «religieux» ? À cette question, une réponse toute prête se tient à notre portée: c'est un penseur dont l'entreprise intellectuelle est nourrie et soutenue par une foi et se comprend elle-même en dernière instance comme l'explicitation d'un contenu religieux positif et déterminé. La foi comme relation fondamentale et le dogme comme matière ultime de la pensée et cadre du discours, tels seraient les deux appuis à l'aide desquels procéderait la pensée dite «religieuse ». Dès lors, pour qui ne reconnaîtrait pas l'un et se saurait ainsi dépourvu de l'autre, pour qui n'avancerait à la lumière — ou dans l'ombre — d'aucun «credo ", la tâche propre du penseur religieux apparaîtra immanquablement comme étrangère, voire - si l'on ne déclare recevables que les données fournies par la raison ou par l'expérience - comme philosophiquement suspecte. On étudiera alors une telle pensée pour son intérêt culturel, historique, esthétique, spirituel même, mais on lui déniera le statut de "philosophique ". Quelle que soit la latitude avec laquelle il faille entendre ici les mots «foi » et «dogme", selon les divers contextes religieux, historiques, linguistiques, etc., il n'en reste pas moins qu'une telle compréhension sommaire passe sous silence le fait premier qui permet de caractériser un penseur comme «religieux». Ce fait tient essentiellement à une expérience en laquelle

* Vincent Giraud est maître de conférences (Hakubi researcher) au Département de philosophie de la religion de l'Université de Kyōto. Il travaille sur la réception du néoplatonisme par les penseurs de l’École de Kyōto. Il a récemment publié (2013) Augustin, les signes et la manifestation, Paris, PUF (Épiméthée) et édité (2012, en collaboration avec Benoît Jacquet) From the Things Themselves. Architecture and Phenomenology, Kyōto, Kyōto University Press/École française d'Extrême-Orient.

(C) Revue Théologiques 2012. Tout droit réservé. 
vacillent les certitudes induites par l'existence courante et, surtout, lors de laquelle l'existence ne se donne plus pour la source ultime et autorisée de ces mêmes certitudes, cessant donc par là de les garantir. Comme l'écrit Nishitani, "nous prenons conscience de la religion comme d'un besoin, comme d'une nécessité pour la vie, seulement lorsque la vie atteint un niveau auquel tout le reste perd sa nécessité et son utilité ${ }^{1}$ » (Nishitani 1982, 3). Cependant, pour ne pas être une simple péripétie au sein d'une existence individuelle et singulière, et comme telle contingente, cette épreuve lors de laquelle tout perd réalité et poids, sens et valeur, doit en outre être imputée à l'existence elle-même, à l'« essence " même de l'existence. L'existence subjective et mondaine se dévoile alors comme le lieu d'une crise et comme cette crise même, dans l'impuissance où elle se trouve de s'assurer par ses seules ressources un statut et une position au sein de l'être. Lorsque la pensée se fait méditation de l'existence comme crise, et que c'est à "autre chose ${ }^{2}$ » qu'à l'existence nue qu'il est demandé de rendre compte de cette détresse, mais aussi d'esquisser l'issue qui conduit hors d'elle, alors la pensée peut être dite " religieuse $^{3}$ ».

De telles considérations préliminaires, aussi hâtives soient-elles, n'ont rien de superflu lorsqu'il s'agit d'aborder la pensée d'un philosophe qui a choisi d'intituler son maître ouvrage Qu'est-ce que la religion ?4 En effet,

1. Nous traduisons à partir de l'anglais. L'ouvrage sera désormais désigné par l'abréviation « $R N »$.

2. Cet «autre chose», comme nous allons le voir, n'est pas pour Nishitani un "principe ", ni une "substance », ni aucune instance objectivable. Il n'en reste pas moins que c'est à une percée hors de la forclusion propre à l'existence subjective - c'est-àdire telle que vécue sur son mode le plus immédiat et le plus ordinaire - qu'invite le philosophe japonais.

3. Voir Greisch $(2002,43)$ : «La "philosophie religieuse” [...] s’intéresse précisément à la manière dont un sujet assume philosophiquement les défis intellectuels et existentiels qui découlent de son expérience spirituelle personnelle. Les Confessions de saint Augustin, la "grammaire de l'assentiment" du cardinal John Henry Newman, la finale de L'Action de Maurice Blondel nous fournissent des échantillons particulièrement éloquents de ce type d'interrogation. " On pourrait ajouter à ces noms illustres celui de Nishitani.

4. Ce sont des raisons éditoriales, et comme telles étrangères à toute considération philosophique, qui ont imposé un changement de titre pour l'édition américaine. On sait par ailleurs, grâce aux informations fournies par le traducteur — dont le travail a été mené de concert avec l'auteur - à quel point Nishitani tenait à la formulation originale du titre et à son énoncé sous forme de question. Voir Van Bragt (cité dans Unno 1989, 7): "The English title became Religion and Nothingness, but I can as well tell you that Professor Nishitani was not happy at all about the change and that it took a few months of diplomacy before he finally consented it. » 
la question se pose d'emblée de savoir en quoi une approche de l'essence de la religion peut soutenir et innerver le questionnement philosophique et, plus radicalement, en quoi la religion peut être dite constituer l'objet — à moins qu'il ne s'agisse de la voie — par excellence de la philosophie. Car telle est bien l'hypothèse à laquelle conduit une prise en compte sérieuse du titre de l'ouvrage, dont on connaît par ailleurs l'ambition et la portée. Car, sous un abord modeste, ce titre dissimule en réalité une entreprise d'envergure. Loin de constituer un thème régional, le questionnement sur l'essence de la religion y sert de point de départ et de fil conducteur à une construction qui, prenant acte de l'effondrement des structures classiques de la métaphysique, leur substitue une conceptualité issue pour une large part de la tradition bouddhiste indienne et extrême-orientale. C'est surtout ce dernier aspect qui a, jusqu'à présent, retenu l'attention des lecteurs occidentaux, lesquels ont principalement concentré leurs analyses sur l'usage nishitanien de la notion de néant ${ }^{5}$. Et cela à très juste titre, tant il est vrai que Nishitani propose une profonde reprise du questionnement métaphysique à partir du néant ou, mieux, de la vacuité (japonais: 空, $k \bar{u}$; sanscrit: śūnyat $\left.\bar{a}^{6}\right)$, qu'il érige en concept clef de sa philosophie et qui provient en droite ligne du bouddhisme le plus ancien. Pour notre part, nous voudrions ici tenter une approche qui, ne prenant pas pour thème la vacuité, y parvient néanmoins et prétend même en atteindre le cœur. Cette voie, que son évidence a parfois conduit à négliger, c'est celle-là même que Nishitani n'a cessé de proclamer comme sienne, conduisant à la découverte de la vacuité et à sa formulation philosophique: c'est celle de la question qui interroge toujours plus avant en direction de la religion et de son essence. En réalité, on peut aller jusqu'à dire qu'une lecture de Nishitani qui prétendrait comprendre son concept de vacuité, en faisant l'économie du «détour» par la religion, se priverait elle-même des moyens d'atteindre ce qu'elle vise. Si l'ouvrage mérite finalement son titre, c'est dans la mesure où, selon Nishitani lui-même, le nouveau dispositif conceptuel élaboré autour du śūnyatā n'a de sens que pour qui se rend capable d'une certaine forme de "conversion ", un décentrement radical de l'existence en direction de la vacuité à partir de laquelle seul se dessine un accès authentique à l'être.

5. Voir, en particulier, Unno (1989), Dallmayr (1992), Elberfeld (2011), Jones (2011), Swanson (1996).

6. Le lecteur trouvera, en fin de volume, un glossaire français-japonais rassemblant les termes clés que l'on rencontre chez Nishitani et certains autres philosophes de l’École de Kyōto. 
On pressent alors que, partant de telles prémisses, une lecture de Qu'est-ce que la religion? gagnera nécessairement à être menée sous la conduite d'Augustin. L'évêque d'Hippone, dans ses Confessions, a uni le plus étroitement qu'il soit possible pensée philosophique et quête religieuse $^{7}$. Plus précisément encore, les Confessions nouent pour la première fois en un seul faisceau trajectoire d'une existence individuelle, approche philosophique de la vérité et découverte du Dieu chrétien. C'est sur le fond d'une telle corrélation que doit être pensé le rapport profond de Nishitani à Augustin. On sait que l'auteur de Qu'est-ce que la religion? n'a cessé de lire et de méditer les Confessions. Quelques références précises en attestent d'ailleurs, au fil de l'ouvrage. Mais ces renvois explicites ne rendent pas justice à l'imprégnation augustinienne dont l'effet se fait sentir bien au-delà de ces quelques citations. Plus essentiellement, c'est un même sol de pensée que partagent l'évêque africain et le penseur de Kyōto. Notons qu'il ne sera pas ici question du christianisme au sens large, dont il est souvent traité au fil des différents chapitres du livre, mais bien de la démarche augustinienne elle-même, dans ce qu'elle a d'original et d'irréductible au message chrétien, ce qui fait d'Augustin une figure unique dans l'histoire de la pensée. Il y a, courant sous les thèses successives de Qu'est-ce que la religion?, aussi discrète qu'une trame, mais aussi solide qu'elle, un dialogue silencieux avec Augustin. Si, comme aimait à le rappeler Heidegger, "un dialogue avec un penseur ne peut porter que sur la "cause" (Sache) de la pensée» $(1996,277)$, c'est ce nœud primitif qu'il importe de mettre au jour dans le rapport que Nishitani entretient avec l'auteur des Confessions. Ce ne sont pas à proprement parler des positions, des thèses ni même des thèmes ou des concepts, que le philosophe japonais retient de la pensée augustinienne, mais, ce qui importe davantage, il en épouse le mouvement parce qu'il en partage la direction et jaillit du même élan. Trame augustinienne plus secrète que tout motif, donc, parce que plus essentielle, et avec laquelle il est question de la cause même de la pensée.

Dans les Confessions, cette cause se déploie selon un triple mouvement, indissociable de l'épreuve que l'existence fait d'elle-même, et au gré duquel elle accède peu à peu à sa propre vérité en Dieu. Le premier moment est celui de la crise: l'ego augustinien se découvre dans son instabilité et

7. Identification exprimée par la formule célèbre de La Cité de Dieu (VIII, 1): «Le véritable philosophe est celui qui aime Dieu (Verus philosophus est amator Dei).» Ou encore, dans le De vera religione: "La philosophie, c'est-à-dire l'étude de la sagesse, n'est pas autre chose que la religion » $(5,9, B A 8$, traduction modifiée). 
son inconsistance, devenant pour lui-même question. Cette crise ne trouve sa résolution que dans une conversion, vigoureux retournement intérieur par lequel le moi ainsi éveillé embrasse l'incommensurable altitude de son ascendance divine. Le retour à Dieu qu'est la conversion ouvre enfin, en même temps qu'à un soi rénové, à un rapport renouvelé avec le réel, et que désigne chez Augustin le mot confession. Or, nous entendons ici montrer que ce schème augustinien fondamental articulant quaestio, conversio et confessio forme précisément la trame privilégiée par Nishitani dans son grand ouvrage.

\section{La destitution de l'ego et l'érosion du monde - Quaestio}

Les Confessions s'ouvrent sur une adresse à Dieu qui est reconnaissance intime d'une inquiétude - au sens fort que revêt ce terme en latin: "Tu nous as faits orientés vers toi (ad te) et notre cœur est sans repos (inquietum) jusqu'à ce qu'il repose (requiescat) en toi (in te) » (Conf. I, 1, 1). C'est ici, dans cette expérience d'une instabilité native du moi humain, qu'il faut chercher la cause de la pensée d'Augustin. Comme telle pourtant, en son pur caractère de fragilité, encore dépourvue de toute attitude par laquelle elle se prendrait elle-même en vue, cette inquiétude ne saurait à elle seule soutenir la recherche. L'immédiateté de l'inquiétude exige la médiation qui saura faire d'elle une authentique question. Si toute pensée véritable jaillit d'une question originaire, il revient à Augustin d'en avoir donné la formulation la plus personnelle autant que la plus radicale.

Par deux fois, dans les Confessions, Augustin parle de lui-même comme de la question (quaestio) qu'il est devenu pour lui-même. Dans les deux cas, celle-ci surgit en un moment qu'on peut à bon droit qualifier de «critique», puisque le premier moment prend place dans la douleur qui suit la mort de l'ami, et que le second s'inscrit dans le contexte d'une analyse des tentations et du tiraillement intérieur que celles-ci entraînent (Conf. IV, 4, 8 et X, 33, 50). Douleur et tentation, toutefois, ne constituent pas de toutes pièces la question. C'est l'inquietudo première qui affleure dans ces moments de crise, elle s'impose sous une forme nouvelle au moi souffrant ou tenté, mais elle avait, d'une certaine façon, toujours été là. Le dévoiement du désir ${ }^{8}$ n'a fait que différer cette exigence en la dissimulant. À travers la question, le moi ne fait plus simplement l'épreuve quotidienne

8. Voir Conf. III, 6, 11: «cum te non secundum intellectum mentis [...] sed secundum sensum carnis quaererem. » 
de sa propre instabilité, mais accède à une vue sur cette dernière qui, en retour, se mue en interrogation. Car, et c'est là le point capital, il ne s'agit pas tant d'une question portant sur le moi que de la question que devient le moi lui-même pour lui-même'. Et ce n'est pas la crise qui fait la quaestio, mais bien qui la révèle, par le brutal dévoilement de la dissemblance et de l'indigence qui structurent le moi.

Le premier passage montre Augustin en proie à la douleur de la perte de l'être cher $^{10}$. Le contexte est ici décisif, car il permet de cerner la teneur de la quaestio:

Cette douleur enténébra mon esprit et partout je ne voyais que mort. La patrie m'était un supplice, la maison paternelle un étrange tourment, tout ce que j'avais partagé avec lui s'était tourné sans lui en torture atroce. Mes yeux le réclamaient de tous côtés, et on ne me le donnait pas (non dabatur), et je haïssais toutes choses (oderam omnia), parce qu'elles ne l'avaient pas et ne pouvaient plus me dire: «Le voici, il va venir », comme quand il vivait et qu'il était absent. J'étais devenu pour moi-même une immense question (factus eram ipse mibi magna quaestio), et j'interrogeais mon âme (interrogabam animam meam): pourquoi était-elle triste, et pourquoi me troublaitelle si fort? Et elle ne savait rien me répondre. (Conf. IV, 4, 8)

La douleur de la perte met au jour le moi comme instabilité. Ne pas se tenir en soi-même apparaît, à la lumière de la disparition de l'ami, comme condition originaire. "Haïr toutes choses", c'est les voir comme pure absence de celui qui est aimé. Cette absence polarisée par l'amour s'affirme comme frustration cuisante ( "on ne me le donnait pas", " elles ne l'avaient pas») manifestant ce qu'Augustin nomme ailleurs la «région d'indigence (regio egestatis)» (Conf. II, 10, 18), autre nom pour désigner la facticité envisagée comme domaine de l'immédiateté dissemblante (regio dissimilitudinis, Conf. VII, 10, 16). Alors surgit la question que le moi est pour soi-même. Être-au-monde et ipséité se soutiennent mutuellement en une commune épreuve, de sorte que le moi s'apparaît devant soi-même: "Et tu me mettais bien en face de moi (et constituebas me ante faciem meam)" (Conf. VIII, 7, 16). Quand s'impose au moi la vision de soi comme énigme, question, problème, le monde a déjà été vécu comme manque dans une

9. Selon les termes de H. Arendt, l'homme est cet étant «qui, introduit dans le monde par création, vit dans une interrogation spécifique sur son être propre " (Arendt 1999, 107).

10. Sur cet épisode, voir Bouton-Touboulic (1999), Marion $(2008,101)$. 
solitude inquiète portée par un amour sans objet ${ }^{11}$. Le « devant soi-même ", vécu dans la douleur et le désarroi du questionnement, est le stade primitif à partir duquel peut s'amorcer une remontée au «devant Dieu (coram Deo $)^{12} »$.

Et c'est bien cette fois devant Dieu que s'effectue à nouveau le devenirquestion du moi dans le présent de l'écriture des Confessions, c'est-à-dire à un stade ultérieur du parcours existentiel. Dans ce second passage, qui fait immédiatement suite à une analyse des tentations auditives, Augustin s'exprime ainsi: «Mais toi, Seigneur mon Dieu, entends, regarde, vois, aie pitié, guéris-moi, toi sous les yeux de qui je suis devenu pour moi-même une question (in cuius oculis mihi quaestio factus sum)! »(Conf. X, 33, 50, traduction modifiée). Pourtant, l'appel à Dieu ne dissipe pas encore la question; il la pose sur une autre base. L'appel est lancé en direction de Dieu, mais pour lui demander aide et secours du fond de la condition de tentation. C'est l'inaptitude à échapper par ses seules forces au règne des tentations - dont Augustin est, du reste, à ce stade, parfaitement conscient, ainsi que du bien qu'il lui faut poursuivre - qui caractérise cet appel du moi. Car savoir n'est pas encore vouloir pleinement et le moi devient question pour lui-même lorsqu'il flotte (fluctuo) en proie à des désirs contraires dans l'éparpillement (dispersio) et l'atermoiement (tardatio). C'est cette fois l'indécidabilité, la difficulté de la décision à partir d'un désir divisé, et donc d'un amour encore imparfait, qui fait la teneur de la quaestio.

Ces deux textes ouvrent donc un double accès au moi envisagé dans sa facticité. Chaque fois, cet accès est ménagé à partir d'une prise en compte de l'amour. Le moi apparaît à lui-même comme incapable de se suffire, c'est-à-dire aussi de se maintenir dans sa suffisance. La structure «questionnante» du moi caractérise sa visée intrinsèquement désirante, mais celle-ci y est renversée en la conscience de l'incapacité de pourvoir à ce désir. Dans le "devant Dieu », qui est comme la scène sur laquelle prend place la quaestio dans le second passage, c'est la conscience de la dissemblance du moi à l'égard de son Créateur qui précipite la crise subjective; ce que confirme un autre texte:

11. La suite immédiate de l'extrait étudié ici montre le jeune Augustin incapable de soutenir la relation à Dieu, car encore captif d'une fausse compréhension de la divinité: "Et si dicebam: "Spera in Deum", iuste non obtemperabat, quia verior erat et melior homo, quem carissimum amiserat, quam phantasma, in quod sperare iubebatur " (Conf. IV, 4, 9).

12. Voir Conf. I, 1, 1: "coram te in confessione». 
Devant toi, Seigneur, ma substance sera comme un rien (Ante te, Domine, tamquam nibil substantia mea), devant toi qui vois cela; et moi, si je le vois, ce n'est que devant toi, et non devant les hommes. Que dirai-je? Quelles paroles pourraient montrer que ce que je suis n'est rien en comparaison de celui qui est (nibil esse quod sum in comparatione eius quod est)? (Enarr. in Ps. 38, 9, nous traduisons).

Alors, le moi que creuse un tel désir sans mesure avec sa propre finitude peut se dire aussi bien "abîme ${ }^{13}$ ". Devenir une question pour soimême signifie alors se connaître inconnaissable, à partir du pressentiment ou de la reconnaissance, en soi, de cet infini qui n'est pas soi. L'inconnaissable que je suis n'est pas dissociable de l'interrogation qui me donne à moi-même.

Il était nécessaire d'établir avec suffisamment de précision le double contexte augustinien dans lequel se fait jour la quaestio afin de pouvoir mesurer ce qu'en retient Nishitani, mais aussi l'inflexion propre qu'il lui imprime. C'est d'entrée de jeu, dès que se trouve défini de façon liminaire le sens à reconnaître à la religion, que s'impose la quaestio. Immédiatement après avoir décrit l'existence se découvrant elle-même sans but ni sens comme lieu d'émergence de la religion, Nishitani précise ainsi son propos: «Lorsque nous en venons à douter ainsi du sens de notre existence, quand nous sommes devenus une question pour nous-mêmes, la quête religieuse s'éveille en nous " $(R N, 3)$. Ainsi, à l'origine même de sa démarche, l'auteur de Qu'est-ce que la religion? place une crise semblable à celle qui déchirait le moi augustinien. Plus frappant encore, c'est en recourant à l'exemple de la perte d'un être cher, dont nous venons d'évoquer le récit tel qu'il figure au livre IV des Confessions, que Nishitani explicite dans cette même page le contenu de la quaestio. Lorsque meurt l'ami avec qui on partageait tout, " toutes ces choses qui tenaient jusqu'alors leur usage d'être pour lui deviennent bonnes à rien» $(R N, 3)$. Quand on se trouve ainsi face à face avec la mort, "l'existence du soi [...] vient saillir sur fond de nihilité ${ }^{14}[. .$. un vide apparaît que rien au monde ne peut combler, un abîme béant

13. «Le profond abîme (grande profundum) qu'est l'homme lui-même! » (Conf. IV, 14, 22); "l'abîme (abyssus) de la conscience humaine " (Conf. X, 2, 2). L'abîme désigne tout ensemble la profondeur insondable échappant à la connaissance et le fond $\mathrm{du}$ précipice en lequel se perd la moralité. La quaestio lie bien ces deux sens, elle qui est toujours vécue dans la douleur de la perte ou l'écartèlement des diverses tentations. Profundum et abyssus permettent donc de préciser le sens de quaestio.

14. Nous traduisons ici «nihility» par «nihilité», afin de ne pas la confondre avec le «néant» conçu ultimement par Nishitani comme vacuité. 
s'ouvre sur le sol même où l'on se tient» $(R N, 3)$. On décèle dans ces lignes bien des accents augustiniens. Nishitani se distingue toutefois de l'auteur des Confessions sur deux points. D’abord, le "pourquoi? " ne s'adresse pas ici à l'âme et aux raisons de sa tristesse, mais se porte aussitôt vers la cause ou la raison de l'existence subjective elle-même. Ensuite, épreuve existentielle de la mort et rencontre ontologique de la nihilité sont immédiatement identifiées. Cette dernière emprunte d'ailleurs clairement sa définition à une détermination de l'existence: "La nihilité renvoie à ce par quoi le sens de la vie perd son sens » $(R N, 4)$. Il n'y a pas ainsi de nihilité séparée de son épreuve par le sujet vivant, de son émergence dans une existence concrète. C'est pourquoi nihilité et question ont finalement la même extension: "Lorsque nous devenons une question pour nous-mêmes et quand se lève le problème de savoir pourquoi nous existons, cela veut dire que la nihilité a émergé du fond de notre existence et que notre existence elle-même s'est changée en un point d'interrogation. » Le texte augustinien se trouve donc doublement radicalisé: une première fois avec l'assignation de l'existence elle-même comme direction du "pourquoi ?" et une seconde fois lorsqu'est faite, de l'absence irrémédiable, une épreuve authentique du rien frappant tout sens de nullité.

Mais, en réalité - et un retour au «mihi quaestio factus sum » tel qu'il apparaît cette fois au livre X nous l'apprend - une telle lecture ne fait que presser le sens ultime de la «question » telle qu'Augustin l'a pensée. Car, pour que le sens de l'existence lui-même soit en suspens, pour que le réel s'absente jusqu'à se montrer partout sous l'apparence de la mort et, finalement, du rien, il faut que soit interrompue la condition en laquelle nous nous tenons ordinairement. Cela peut advenir, comme au livre IV, avec la mort de l'ami, mais aussi, plus explicitement encore, lorsqu'est brutalement mis à nu le sol même que l'existant avait jusqu'alors considéré comme le sien et comme le mode même de son rapport à la réalité. Chez Augustin, cette condition ordinaire, identique à la facticité même de l'ego, prend le nom de «tentation (tentatio $)^{15}$ ». Par ce mot est désignée la dispersion de l'amour propre à l'homme pécheur, dont le désir se porte vers les créatures et vers soi-même, dans un mouvement ultimement imputable à

15. À Heidegger revient encore le mérite d'avoir reconnu dans la tentation non pas un simple état accidentel ou contingent du moi, mais un véritable existential. Voir la lecture du livre X des Confessions dans la Phänomenologie des religiösen Lebens (GA 60), particulièrement p. 248 ("[Die tentatio ist] kein Geschehnis, sondern ein existenzieller Vollzugsinn, ein Wie des Erfahrens») et p. 256 ( Inwiefern die tentatio ein echtes Existenzial.", souligné dans le texte). 
l'orgueil (superbia). Le livre $\mathrm{X}$ analyse tour à tour les tentations sensibles, les voluptés, les curiosités, ainsi que celles relevant directement de l'amour de soi. Au final, cet examen introspectif donne ainsi le tableau, non d'une série d'infimes accidents parsemant la vie personnelle d'Augustin, mais bien d'une condition universelle, propre à la vie humaine elle-même dont la tentation constitue alors le ressort permanent ${ }^{16}$. Pensée à partir du plaisir de l'ouie, la quaestio dévoile un moi rivé à soi-même par l'attache de la tentatio et incapable de se hausser avec liberté et aisance jusqu'à une forme plus haute de jouissance qui n'aurait plus le moi pour pôle et pour centre: «Et la vie heureuse, la voilà, éprouver de la joie pour toi, de toi, à cause de toi (gaudere ad te, de te, propter te)» (Conf. X, 22, 32). La «question» est celle d'un moi qui ne peut échapper à soi-même.

Le devenir-question prend ainsi le sens d'une épreuve des limites mêmes de l'ego. Cette attache qu'Augustin identifie comme tentation, Nishitani la pense comme "mode d'être autocentré", "moi enfermé en soi-même» ou «attachement à soi» $(R N, 14)$. À cet égard, la question que le moi devient pour soi-même constitue une première brèche dans le mode d'être ordinaire du moi. Car, en devenant question, en se vivant dans le «Grand Doute », le moi fait l'expérience de la nihilité comme de son sol le plus véritable, il accomplit un pas en direction d'une rupture de la chaîne qui le retient à soi-même. Le réel environnant n'est plus alors envisagé comme pour le moi, mais comme dénué de sens et de valeur, sans fondement solide. Le moi lui-même se découvre sans subsistance ni but, privé douloureusement de ce qui faisait son milieu ordinaire. Exprimé formellement, cela signifie que

Le plan de la nihilité [...] apparaît au point où se rompt tout l'enchevêtrement du subjectif et de l'objectif; sur le plan de la nihilité, tout ce qui est communément dit exister ou être réel sur les terrains de la sensation et de la raison est démasqué comme ayant pour sol la nihilité, comme dénué, depuis le début, d'un enracinement. $(R N, 122)$

C'est la raison pour laquelle, sur un tel plan, «il n'est même plus clair de savoir ce que je suis moi-même» $(R N, 124)$ : l'émergence de la nihilité dans le devenir-question entraîne cette conséquence que «l'existence des choses et du moi sont toutes deux transformées en quelque chose d'absolument incompréhensible, dont nous ne pouvons plus dire ce qu'elle est » $(R N, 136)$. Mais il faut ici prévenir une équivoque. Contrairement à toutes

16. Pour une analyse de la tentation en ce sens, voir surtout Marion (2008, $\mathbb{2} 23)$, «La tentation et le fait de soi », p. 205-213. 
les formes classiques de scepticisme, le Grand Doute, tel que le conçoit Nishitani, ne met pas en cause l'existence des choses, ce qui supposerait encore qu'il y eût, d'une manière ou d'une autre, présents à la « conscience », un sujet doutant, des objets dont on douterait, le tout articulé autour d'un concept d' "existence » laissé lui-même ininterrogé. La "question », au contraire, «en dévoilant dans la nihilité l'existence même des choses, dévoile l'existence elle-même comme un véritable "doute» », en même temps que celle des choses, c'est «l'existence de celui-là même qui questionne au sujet de l'existence qui se transforme en une question" (RN, 111).

La reprise nishitanienne de la quaestio augustinienne, on le voit, ne va pas seulement jusqu'à frapper toute existence, c'est la catégorie même d'existence - en toutes ses acceptions, tant "existentielles » que strictement ontologiques — qu'elle rend impuissante et caduque : "Si l'existence est transformée en une question, alors son dévoilement dans la nihilité ne peut pas fournir un point de vue [adéquat] pour résoudre cette question » et «l'ontologie doit traverser la nihilité en direction d'un champ entièrement nouveau, différent de ce qu'elle a connu jusqu'ici ${ }^{17}{ }^{\prime}(R N, 112)$. En effet, le moi faisant dans la question l'épreuve de la nihilité ne peut paradoxalement pas s'affranchir de l'existence que son expérience même récuse: la nihilité, par son déni même - ce qui en fait un concept «purement négatif (antithétique) " et une épreuve "autocontradictoire » $(R N, 137)$ vécue dans le plus douloureux et déchirant des paradoxes - ne cesse d'en appeler à une existence positive, contre laquelle elle est tout entière bâtie. Il n'y a de nihilité éprouvable et pensable que par rapport à une existence, même destituée. État intenable pour le moi se vivant soimême et toutes choses comme question, la nihilité est aussi du même coup, pour le philosophe, un concept qui, au-delà de son rôle critique, se révèle finalement impraticable. Nulle part davantage qu'ici, sans doute, ne se confondent aussi étroitement les deux aspects propres à toute philosophie religieuse : l'existant aux prises avec le Grand Doute y est indissociable de l'entreprise propre au philosophe de "dépasser le nihilisme ». L'issue recherchée vaut identiquement sur le plan de la vie individuelle et sur celui de la pensée méditante, car ni l'une ni l'autre ne peuvent s'établir dans la nihilité. Conformément au caractère de crise que revêt la "question", «le caractère de la nihilité est essentiellement transitif»(RN, 137). Quel est alors ce mouvement qui, loin de rétablir dans ses anciens droits l'existence

17. Cela rend nécessaire "un tout autre concept d'existence» $(R N, 128)$. 
désormais dessillée, saura franchir l'abîme ouvert par la quaestio et, par là, se ménager un nouvel accès au réel ? À cela, tout l'ouvrage de Nishitani constitue un essai de réponse: "Le point de vue de la nihilité vaut purement comme l'indication de l'inéluctable exigence d'une conversion» $(R N, 112)$.

\section{L'appel du Proche - Conversio}

Contrairement à l'usage qui a fini de nos jours par prévaloir, la «conversion» ne vaut pas primitivement comme adhésion croyante à un principe divin. Elle est avant tout à envisager comme un renversement ou retournement (con-vertere) du moi lui-même. Et c'est seulement à partir de ce premier sens que peut se concevoir la conversion religieuse dans son affirmation d'une foi. Chez Augustin, ces deux aspects s'articulent étroitement, et ce n'est qu'en marquant les étapes d'une métamorphose de l'ego que les Confessions retracent le chemin qui conduit à la foi. Le motif de la conversion, du reste, notons-le d'emblée, n'a rien de spécifiquement augustinien: d'abord biblique ${ }^{18}$, il se conjugue chez les Pères aux concepts issus du platonisme et du néoplatonisme (metanoia, epistrophè) ${ }^{19}$ et acquiert une dimension plus populaire dans ces premiers siècles du christianisme, avec l'injonction de se convertir à la foi nouvelle et d'abandonner les dieux du paganisme. À partir de ce contexte, l'originalité augustinienne est d'enter la conversio sur la quaestio, d'en faire un impératif issu de l'inquiétude travaillant intimement la créature humaine. Comme telle, elle ne relève pas tant d'abord de l'esprit et de son intelligence que du cœur et de son amour; elle est conversio cordis. Si la conversion procède à une transformation en profondeur du moi, c'est ainsi parce qu'elle est la réponse au premier mouvement d'orgueil qui inaugure la condition de pécheur et par quoi Adam s'est détourné de Dieu (a-vertere, aversio) pour tourner son amour vers soi-même et vers les créatures ${ }^{20}$. La conversion s'impose à partir de l'état

18. Voir l'article «Conversion» dans Lacoste (1998).

19. Voir les pages que Pierre Hadot a consacrées au motif de la «conversion", reprises dans Plotin, Porphyre: Études néoplatoniciennes, Paris, Les Belles Lettres, 2010.

20. Lib. arb. III, 24, 72: "Et d'où vient ce détournement (aversio), sinon de ce que celui dont Dieu est le bien, veut être pour lui-même son propre bien, comme Dieu l'est pour lui-même?" Voir aussi, par exemple, Imm. anim., 19: "Sapientiam vero, quia conversione habet [i. e. animus] ad id ex quo est, aversione illam potest amittere. Conversioni namque aversio contraria est.»; Div. quaest. ad Simpl. I, qu. 2, 18: «Est autem peccatum hominis inordinatio atque perversitas; id est, a praesentiore Conditore aversio, et ad condita inferiora conversio." 
de tentation comme mode d'être ordinaire, lorsque celui-ci traverse la crise qui le révèle comme quaestio. Par elle, nous nous détournons de ce qui nous avait d'abord captivés, nous haussant par là à un amour meilleur: l'homme "change en mieux lorsque peu à peu il détourne (avertit) son intention et sa gloire des choses terrestres, celles qui apparaissent dans ce siècle, et qu'il se retourne (convertit) vers les choses supérieures et intérieures» (Epist. 55, 9). Ces lignes l'indiquent avec netteté: la conversion opère un décentrement $d u$ moi, dont le centre de gravité bascule alors, se situant non plus dans les choses et dans la jouissance égoïste qu'en tire ou en escompte le moi, mais dans l'amour porté à Dieu seul (que désignent ici les superiora atque interiora). Quoiqu'échappant à la contingence par son ancrage dans la condition de tentation, ce mouvement n'en est pas pour cela rendu nécessaire ni automatique. Au contraire, Augustin narre avec détails les longs efforts et les multiples rechutes dont s'accompagne la douloureuse transformation du moi. La conversion prend de la sorte place au sein d'une dramatique de la décision lors de laquelle l'existant rompt avec ses anciennes attaches et l'ego, se décentrant de son pôle mondain, se recentre en Dieu. Par là, l'enjeu est bien celui de la reconquête d'une stabilité que l'existence subjective se sait désormais, comme quaestio, impuissante à garantir: "En toi-même pourquoi te tiens-tu et ne te tiens-tu pas? (quid in te stas et non stas?) ", s'exhorte ainsi Augustin, dans cette lutte "de [lui]-même contre [lui]-même (de me ipso adversus me ipsum)» (Conf. VIII, 11, 27). L'adoption de Dieu comme centre ne s'impose que dans la mesure où il est pris peu à peu conscience du fait que lui seul pourvoit à l'assise dont est par elle-même privée toute existence: "Alors je serai stable et solide en toi (Et stabo atque solidabor in te)» (Conf. XI, 30, 40).

Ainsi, lorsque Nishitani soutient que «la conversion fondamentale en toute vie est suscitée par l'ouverture de l'horizon de la nihilité à partir du sol même sur lequel se tient la vie » et que "ce n'est pas là autre chose qu'une conversion à partir du mode d'être autocentré (ou humainement centré) » $(R N, 4)$, il fait sienne une profonde intuition augustinienne. Un tel décentrage est induit par la question que le moi devient pour lui-même comme émergence de la nihilité. En même temps que de l'effondrement du monde, dont s'est absenté tout sens, toute valeur et toute subsistance, le moi fait l'expérience de son inconsistance en tant que leur possible foyer. $\mathrm{La}$ "question" n'est pas encore la conversion, mais elle l'appelle, en la rendant indispensable. La destitution du moi, comme centre, ruine la structure de l'ego comme creuset en lequel se recueille le monde et celle du monde comme «en-face» offert à un ego. La question qu'est devenu le moi 
réclame l'acte par lequel sera abandonnée la posture de l'ego, mais elle n'en est pas encore l'accomplissement. La conversion seule l'opère, en prenant résolument appui sur l'abîme ainsi ouvert. Si l'émergence de la nihilité a révélé la fausse prétention du moi à s'ériger en centre de l'ordonnancement du réel et à tout envisager à partir de ce centre, si rien ne demeure du sujet ni de ses objets, reste alors cette issue qui consiste à vivre sur un mode qui ne répondrait plus du tout des catégories de sujet et d'objet ni même de toute $e k$-stase qui prétendrait en rendre compte ${ }^{21}$. Le décentrement radical auquel appelle Nishitani ne quitte pas le lieu du moi pour l'attacher à quelque autre chose qui lui serait extérieur, il dénie toute extériorité — par défaut d'intériorité subjective qui lui ferait pièce — et conteste ce partage même de l'intérieur et de l'extérieur. Certes, l'expérience commune m'apprend bien que «je» ne suis pas «les choses» ni «autrui», mais qu'advient-il de cette certitude, dès lors que se trouvent supprimés les concepts courants de «moi », de "choses» et d' "autrui»? Plus positivement, quel sol, commun au moi, aux choses et à l'autre humain, se dévoile-t-il sur le fond de notre commune mise en échec dans le devenir-question? La conversion réalise ainsi que la nihilité n'est pas l'absentement du réel, mais bien son insistance, sur un mode toutefois qui demande à ce qu'il soit vécu comme unité transcendant les catégories ordinaires en lesquelles la facticité avait cru trouver un abri et fait comme «sujet» l'expérience d'un «monde». La question ne ruine pas le réel, mais seulement sa saisie subjective-objective et, en dernière instance, extatique. Le décentrement remonte au plan que le moi et le monde avaient en partage, plan qu'ils scindaient abstraitement en le recouvrant.

Sur le terrain propre du moi, cela signifie la conversion à ce qui, en moi, n'est pas identifiable comme ego ou "sujet». Pour exprimer ce fait, Nishitani se heurte évidemment aux limites du vocabulaire et à ses partages calqués sur l'expérience du mode d'être ordinaire. Ce qu'il a en vue est bien une forme de "subjectivité » (il ne renonce pas au terme), mais qui ne relèverait plus d'un quelconque mode d'être égoïque ou "personnel». Il s'agit d'accomplir - ou, plus exactement, ce qui n'est guère plus aisé, de se livrer à — «la négation de cette subjectivité que la personne s'assigne à elle-même lors de sa saisie par elle-même sur le mode de l'auto-centrage ». Cette négation, précise aussitôt le texte, "a le sens d'une conversion [qui opère] à l'intérieur de la personnalité fermée sur soi »; car, «lors de cette négation, la personne est brisée de l'intérieur et le moi personnel se

21. Sur l'ekstasis au sens de Heidegger, voir RN, 68. 
découvre comme subjectivité en son sens élémentaire (elemental), comme ipséité (selfhood) véritable» ( $R N, 72)$. Voilà en quoi consiste finalement la "conversion existentielle»: elle est un «changement de cœur» redécouvrant ce qu'il y a de "plus proche» $(R N, 70)$, que le mode d'être ordinaire subjectif-objectif à partir duquel procède toute conscience ne pouvait que dissimuler et que la quaestio révélait brutalement sous les traits angoissants de la nihilité. "Le "rien du tout" se tenant derrière la personne éclate au grand jour du côté du moi, du moi originel » (RN, p. 70). Par là, il apparaît qu'en son mode d'être originel, le moi a la consistance, non d'un "sujet» au sens classique, mais d'un élément, celui qu'il partage avec toutes choses. Dans «la vraie Forme de sa talité », le moi est «impersonnel ${ }^{22}$ » $(R N, 74)$, il s'apparaît comme un avec les choses et "la conversion qui a lieu quand on dépasse le plan de la nihilité [...] signifie que toutes choses, en leur talité, sont rassemblées en une unité » $(R N, 143)$.

$\mathrm{Si}$, sur le terrain du mode d'être ordinaire, toute proximité s'éprouve pour nous comme contact avec un étant - que celui-ci soit physique, sensible ou intellectuel, rapport à une extériorité ou saisie de soi par soi sur le mode de l'ego - il faut alors reconnaître une proximité plus essentielle lorsque se trouve rompu ce mode personnel de saisie. En son sens véritable, la conversion nishitanienne constitue une réponse à l'appel du Proche entendu dans la quaestio. Ce Proche nomme l'insistance du réel une fois ruinée la structure imposée par la conscience. On ne peut plus le dire chose, ni ego, ni autrui, ni substance, toutes catégories ayant révélé dans la nihilité leur commune inconsistance. Mais ce «rien" de la nihilité doit lui-même être dépassé, au nom précisément de ce qui insiste en elle et dont le fait de n'être plus rien qui se prête à une objectivation ni à une subjectivation ne saurait en aucun cas suffire à déclarer la pure et simple absence. Comme «conversion absolue» (RN, 138), c'est-à-dire ne se connaissant pas d'autre terme que celui dont elle s'affranchit, la conversion à laquelle invite Nishitani est une conversion au Proche. Mais par là se trouve du même coup abolie toute distinction du lointain et du proche, puisque, sur le terrain ainsi conquis, «l'atteinte du lointain n'est rien de moins que le Proche absolu »: ce terrain est celui de la vacuité, ou śūnyatā $(R N, 138)$. La vacuité n'est pas le contraire de l'être, mais nomme l'insistance ordinairement dévolue à l'être une fois disqualifié son mode ek-statique de déclosion au moi. Un Proche, autrement dit, qui ne s'abrite dans aucune

22. Faute de solution satisfaisante en français, nous rendons suchness (japonais: 真如, shinnyo; sanscrit : tathatā) par talité. 
Erschlossenheit, et qui serait bien plutôt à saisir, selon Nishitani, comme la condition profonde de cette dernière ${ }^{23}$.

La destitution du soi dans la nihilité et sa transcendance dans la vacuité entrent en consonance cette fois avec une autre thèse fondamentale de l'augustinisme et qui en est même devenue comme l'emblème: "Mais toi tu étais plus intérieur que le plus intérieur de moi-même, et plus haut que le plus haut de moi-même. (Tu autem eras interior intimo meo et superior summo meo) " (Conf. III, 6, 11). On aura reconnu, dans cette phrase célèbre, une transposition des superiora atque interiora vers lesquelles devait se tourner la conversion. L'articulation des comparatifs (interior, superior) et des superlatifs (intimo, summo) ne nomme pourtant pas une présence dont on pourrait s'emparer sans reste, mais le mouvement ascendant d'une remontée vers le Verbe intérieur. Exploitant cette découverte du livre IV, la partie du livre X qui traite de la mémoire se laisse ainsi lire comme une remontée transcendante (qui d'ailleurs se dit au futur: "transibo...») au cœur de l'ego vers ce qui, en lui, n'est plus lui, mais le constitue $^{24}$. Comme on le sait, contrairement à ce que fera la tradition issue de Denys (de Jean Scot à Nicolas de Cues, en passant par Eckhart), à aucun moment Augustin ne va jusqu'à qualifier de «néant » - fût-il «suressentiel» (byperousios) — le Dieu ainsi découvert au-delà de toute saisie intellectuelle. Le terme atteint, lors de cette ascension intérieure, n'en est pas pour cela moins décisif: "Où donc t'ai-je trouvé pour te connaître, sinon en toi au-dessus de moi (in te supra me)? Et nulle part, aucun lieu (nusquam locus); nous nous éloignons, nous nous approchons, et nulle part, aucun lieu» (Conf. X, 26, 37). L'identification du lointain et du proche dans l'absence de lieu (nusquam locus) conquise au terme de la transcendance de soi (supra me) s'exprime comme accès au non-lieu divin (in te).

23. Le rapport de Nishitani à Heidegger mériterait d'être précisé et discuté, mais un tel développement ne peut trouver sa place dans ces pages. Disons seulement que la facticité du Dasein comme ouverture à l'être est envisagée par Nishitani dans la ligne de sa critique du mode d'être propre à la conscience. Elle en représente un plan plus profond, mais que Nishitani ne croit pas originaire.

24. Augustin commence par dépasser sa propre puissance vitale ("Transibo vim meam», 7,11), puis la mémoire en son sens inférieur ("Transibo et hanc vim meam, quae memoria vocatur, transibo eam, ut pertendam ad te, dulce lumen.»17, 26). Et ce mouvement de transcendance une fois accompli ("Transcendi enim partes eius, quas habent et bestiae, cum te recordarer.»), dépassant même "le siège de son esprit lui-même», il parvient au Dieu éternel ("tu autem incommutabilis manes super omnia») qui habite sa mémoire ( "et dignatus es habitare in memoria mea ", 25, 36), tout en se situant au-dessus d'elle («in te supra me») $(26,37)$. 
C'est au cœur même du soi comme abîme de la divinité ouvrant en soi à un au-delà de soi que ce dernier trouve son sol ultime, irréductible à l'ego et à ses prises. Par la conversion, la divinité s'offre donc comme Proche absolu: "Celui qui a créé est plus proche de nous que la multitude des choses qui ont été créées (propinquior nobis est qui fecit, quam multa quae facta sunt)»(Gen. ad litt. V, 16, 34). Nishitani, reprenant alors à son compte l'interior intimo meo, en fait le mode propre de donnée de la vacuité: "Le śūnyatā représente un champ absolument transcendant et, dans le même temps, un champ qui n'est pas situé sur la face éloignée d'où nous nous trouvons, mais sur notre plus proche à-côté, plus proche que nous ne sommes nous-mêmes pour nous-mêmes ${ }^{25}$ " (RN, 91). Le Dieu d'Augustin, "vie de notre vie ${ }^{26}$ ", trouve dans le discours nishitanien sa transposition comme vacuité, laquelle est "plus proche que nous-mêmes et que notre propre vie telle que nous l'envisageons ordinairement dans l'ici-et-maintenant» $(R N, 90)$.

On dira avec raison qu'il y a loin du Dieu chrétien à la vacuité bouddhique et qu'un tel concours de vue entre Augustin et le penseur japonais pourrait bien masquer des disparités plus grandes encore. Cela est sans réplique. Au-delà de cette évidence, cependant, et plutôt que de comparer terme à terme de trop vastes entités ("Orient» et "Occident ", christianisme et bouddhisme...) il peut s'avérer fructueux de saisir en quoi la reprise philosophique contemporaine par Nishitani de la terminologie bouddhique puise à la source augustinienne pour en infléchir le sens d'une manière inédite. Et c'est bien ainsi, en effet, que les choses se passent dans le cas de l'identification nishitanienne de l'interior intimo meo comme plan du śūnyatā. La vacuité se découvre ici par le chemin d'un dépassement de l'ego à partir de la quaestio et de la conversio.

Les développements qui précèdent, en précisant le mouvement d'une transcendance du soi, auront néanmoins pu donner l'impression d'une exténuation extrême du réel ainsi atteint, le Proche ne paraissant devoir s'avancer que dans l'air raréfié d'une expérience quasi "mystique ». C'est ce malentendu qu'il convient à présent de dissiper, en montrant que, pas davantage que celle d'Augustin, l'entreprise nishitanienne ne vise à nous faire rompre avec la réalité empirique. Tout au contraire, le Proche comme

25. Voir aussi, par exemple, p. 97-100, et p. 130: «Le champ du śūnyatā comme proximité absolue [...] plus proche à-côté que nous ne le sommes nous-mêmes [pour nousmêmes]».

26. Conf. X, 6, 10: «Mais ton Dieu est plus encore pour toi [que ton âme], c'est la vie de ta vie (tibi vitae vita).» 
subjectivité élémentale ne désigne pas autre chose qu'un contact restauré avec le réel.

\section{Le plan du Réel - Confessio}

La conversion augustinienne ne s'achève pas dans un déni du monde, elle aboutit bien plutôt à l'envisager nouvellement comme création de Dieu. Il ne faudrait pas toutefois réduire platement cette saisie à une position théorique, et comme telle contestable par l'intelligence, affirmant que "le monde a été créé par Dieu ", sous peine de se priver par là de tout l'acquis de la conversion. Celle-ci, plutôt que de conduire à une vérité nouvelle ${ }^{27}$, ouvre à une nouvelle manière de vivre la vérité. Au in te supra me du livre $\mathrm{X}$ répond ainsi la phrase du livre XI, dont nous n'avons cité plus haut que le premier membre: "Alors je serai stable et solide en toi, dans ma vraie forme, ta vérité (in forma mea, veritate tua)» (Conf. XI, 30, 40). La conversion, en même temps qu'elle restaure le moi dans sa "vraie forme ", le rétablit sur le sol de la vérité dont il s'était retranché du fait de son aversio, qui le livrait aux diverses tentations. La reconnaissance de Dieu par le sujet converti - à la fois comme connaissance nouvelle, retrouvailles, et comme témoignage de gratitude s'incarnant dans la louange — caractérise la confession augustinienne ${ }^{28}$. Le dévoilement de l'interior intimo meo n'a au fond pas d'autre ressort, ce que révèle négativement le refus de la confession des péchés: "Qu'y aurait-il en moi qui te serait caché (occultum) même si je refusais de te le confesser? Car c'est toi qu'à moi-même je cacherais (absconderem), non pas moi-même à toi» (Conf. X, 2, 2). Le refus ou l'ignorance de la confession équivaut à un voilement de Dieu à soi et bloque toute transcendance du moi, seule capable de rétablir ce dernier dans sa «vraie forme». Mais la confessio peccatorum se double d'une confessio laudis ${ }^{29}$ et c'est alors, dans la louange rendue au créateur, tout l'apparaître même de soi et du monde qui s'en trouve intégralement renou-

27. Immédiatement après la découverte de Dieu «au dessus de [lui] » et en «aucun lieu », Augustin déclare: "Ô vérité, tu sièges partout pour tous ceux qui te consultent [...] Bien tard je t'ai aimée (sero te amavi), beauté si ancienne et si nouvelle, bien tard je t'ai aimée! ( Conf. X, 26, 37-27, 38).

28. Pour les divers aspects liés à ce terme pivot de l'augustinisme, on lira les pages que lui consacre Chrétien (2002, 121-135).

29. Sur la confessio laudis distinguée de la confessio gementis ou confessio peccatorum, voir les analyses de Marion (2008, 50-59) et les extraits cités. 
velé. Au moi confessant son créateur répond une création elle-même confessante:

Et qu'est-ce que cela [i.e. Deus meus]? J'ai interrogé la terre et elle a dit: «Ce n'est pas moi.» Et tout ce qui est en elle a fait le même aveu. J'ai interrogé la mer, les abîmes, les êtres vivants qui rampent. Ils ont répondu: «Nous ne sommes pas ton Dieu; cherche au-dessus de nous. " J'ai interrogé les brises qui soufflent; et tous les espaces aériens ont dit avec ceux qui les habitent: "Anaximène se trompe; je ne suis pas Dieu. » J'ai interrogé le ciel, le soleil, la lune, les étoiles: "Nous non plus nous ne sommes pas le Dieu que tu cherches", disent-ils. Et j'ai dit à tous les êtres qui entourent les portes de ma chair: «Dites-moi sur lui quelque chose.» Ils se sont écriés d'une voix puissante: "C'est lui qui nous a faits. " Mon interrogation, c'était mon attention (intentio); et leur réponse, leur beauté. (Conf. X, 6, 9)

Tout différent de celui qui s'offrait au moi dans l'état de tentation, le monde qui se révèle dans la confession à laquelle aboutit la conversion proclame et loue son auteur. Toute chose n'est plus vue comme une occasion de désirer, mais comme une expression de l'amour divin, suscitant en retour la réponse de l'amour humain. Le décentrement du moi, nous l'avons dit, tient d'abord pour Augustin à une conversion du désir, mais il faut à présent s'aviser du fait que le rapport au réel en ressort à son tour entièrement renversé. Ce qui sollicitait le moi — ainsi les voluptés, qui murmuraient à son oreille comme de «vieilles amies» tentant de le retenir (Conf. VIII, 11, 26) - fait à présent retentir une voix tout autre, qui ne parle pas en direction du moi, mais de Dieu. Pourquoi alors cette voix n'était-elle pas entendue auparavant? "Pourquoi [cette beauté des créatures] ne tient-elle pas à tous le même langage» et est-elle «muette pour l'un» tandis qu' "elle parle pour l'autre» ? C'est que seul l'entend celui qui se tient dans la vérité atteinte par la conversion: «Elle parle pour tous, mais ceux-là seuls comprennent qui, accueillant la voix au-dehors, audedans la comparent avec la vérité (Conf. X, 6, 10).» Or il est un mot qui, chez Augustin, désigne tout ensemble Dieu, l'Univers créé envisagé comme profération divine et sa présence en l'homme comme vérité aimante, c'est celui de Verbe ou de Parole (Verbum). La confession est l'habitation concrète, auditive et vocale, du Verbe par la créature. Par la confession, le moi aborde le monde en vue de Dieu, les trois termes trouvant dans le Verbe leur sol commun et l'élément de leur infrangible union. Seul Dieu 
est le Verbe, mais le moi le retrouve en soi comme raison et comme amour ${ }^{30}$ et le monde l'exprime en tant qu'il est sa création (Jean 1,3). Confesser revient donc à participer pleinement au Verbe: toute chose s'y révèle sous sa «vraie forme", elle aussi, parce que vue non plus du point de vue de l'ego et de ses attentes, mais du point de vue qui ne retient d'elle que sa beauté de chose créée et en rapporte la louange à son auteur. Le réel se donne ultimement pour Augustin comme «Verbe», lequel en constitue la «Forme $e^{31} »$.

Ce plan du Verbe comme habité par la confession semble avoir profondément marqué Nishitani. Si sa terminologie ne fait pas appel aux notions de "Verbe» ou de "confession", qui relèvent directement de la spiritualité et du vocabulaire chrétiens, toute sa pensée est tendue vers le décèlement de la Forme ainsi que du plan sur lequel celle-ci se laisse appréhender. Il n'y a d'ailleurs rien chez Nishitani qui s'apparente à une confession, sauf à considérer - ce que nous faisons ici - que l'enjeu philosophique de la confession consiste pour l'essentiel dans l'instauration d'un plan d'existence accomplissant une restauration du réel pour qui en fait l'épreuve. L'émergence de la nihilité dans la quaestio avait ruiné la chose dans sa vertu de présence disponible et subsistante pour un ego; l'insistance de la vacuité dans la conversio dispose le champ d'une "ouverture absolue» $(R N, 105)$ en laquelle toute chose peut désormais apparaître, non pas selon la vue que porte sur elle un ego, mais à partir du sol sur lequel elle-même se tient. Ce sol originaire n'est autre que ce qui a été reconnu plus haut comme "moi élémental», et c'est en effet tout un que de réaliser la Forme du soi et d'accéder de plain-pied à la Forme de la chose: "Nous ne pouvons entrer en contact avec nous-mêmes que par un mode d'être qui nous met en contact avec les choses du milieu de ces choses elles-mêmes» $(R N, 10)$. Et réciproquement, «la Forme véritable de la talité signifie une rupture à l'égard de toute représentation ou pensée et n'admet pas de saisie par un $e g o »(R N, 107)$. La destitution du sujet est identiquement celle de l'objet, la neutralisation de la conscience - et avec elle de toute intentionnalité — s'accompagne de la disqualification du phénomène

30. Voir la définition donnée dans La Trinité, où le verbe intérieur à l'homme est finalement défini comme "connaissance qui s'accompagne d'amour (cum amore notitia) » (Trin. IX, 10, 15).

31. Voir De vera religione 43, 82: «Ce qui vient en premier, en effet, c'est la Forme de tous les êtres (forma omnium), parfait accomplissement de l'unité dont elle procède; et tous les autres êtres, qui n'existent que dans la mesure de leur ressemblance à l'Un, sont faits par cette forme» (trad. J. Pegon, BA 8). 
conçu comme visée et contenu de conscience. Mais une telle attitude, qui semble prendre à revers la structure même de notre présence à nous-mêmes et au monde est-elle seulement possible? Oui, selon Nishitani, car la conscience même repose sur une non-conscience plus élémentaire, en laquelle consiste originairement son contact avec soi et avec les choses: "La conscience est originellement vacuité [...] il y a une non-conscience à la base de toute conscience, quoique pas dans le sens de ce qu'on nomme “inconscient” » $(R N, 153$, souligné dans le texte). Si la conscience consiste à se saisir soi-même comme ego, alors il faut nommer «non-conscience » élémentale ce qui appert lorsque le travail de la conscience est suspendu. Et de même, quand les choses sont connues dans leur pur être propre, quand a été interrompu et levé le rapport qui nous lie à elles comme à des étants étendus devant, alors on peut parler d'un «savoir du non-savoir»:

C'est le point en lequel le moi est vraiment sur son sol propre (on its own home-ground). Ici, les plantes et les arbres ont pénétré jusqu'au fond pour être eux-mêmes; ici les tuiles et les pierres sont complètement tuiles et pierres; et ici, aussi, en identité avec tout, le moi est radicalement lui-même. C'est là le savoir du non-savoir, le champ de la vacuité elle-même. ( $R N, 110)$

Le moi n'est vraiment le moi que quand il fait un avec les choses, qu'il «devient les choses mêmes qu'il regarde» $(R N, 9)$. En contexte augustinien, on pourrait dire que le moi n'est vraiment le moi que quand il se transcende de façon à se mettre au diapason de la création en prenant part à l'universelle confessio laudis. Alors, la créature est vue telle qu'elle est: qu'on la juge belle ou laide sur le plan de la perception ordinaire, sur le plan de la confession elle est toujours belle, parce que créée. La Forme est par essence "forme belle (forma formosa) », comme le dit une remarquable formule de La Trinité (Trin. XV, 8, 14). Nishitani, lui, n'insiste pas tant sur le caractère de beauté à reconnaître à la Forme de la chose et du moi, sur le plan de la vacuité, que sur leur talité, leur irréductible identité dans le mode singulier et authentique de leur apparaître actuel : "La vraie réalité du moi et de toutes choses, en laquelle tout est présent exactement comme il est " $(R N, 21)$. Mais, à bien considérer les choses, l'écart n'est pas si grand entre talité et beauté. Les accents dont sont porteuses certaines descriptions nishitaniennes ne peuvent tromper: l'arbre et la pierre, l'oiseau et le bambou dont éclate la présence singulière et dont le moi partage le libre essor sur le plan de la vacuité sont évidemment de ces visions propres à séduire le penseur japonais. Et, pour Augustin, la créature n'est belle que parce qu'elle est vue telle que Dieu l'a faite et voulue dans l'éternelle Forme 
de son Verbe. Beauté et talité peuvent enfin être rapprochées parce que toutes deux désignent la manifestation au sens le plus essentiel.

Augustin parle de "manifestatio» essentiellement en deux cas: d'abord pour désigner la vision directe de Dieu réservée aux élus, ensuite pour qualifier la venue du Christ dans la chair; toujours, donc, quoiqu'à deux niveaux bien distincts, il s'agit d'une vision du Verbe ${ }^{32}$. La beauté que découvre le moi confessant appartient ainsi de plein droit, même si c'est d'une manière limitée par sa condition de créature incarnée et de pécheur, à la manifestation dont elle représente à ce titre un mode dérivé. Quant à Nishitani, c'est la manifestation qui fournit la clef d'une juste compréhension du śūnyatā tel qu'il l'entend (bien mieux, selon nous, qu'une confrontation avec la plupart des concepts occidentaux de "néant», qui ne peuvent en ceci qu'égarer la compréhension): "Le śūnyatā est le point auquel nous devenons manifestes en notre propre talité [...] et, en même temps, c'est le point en lequel toute chose autour de nous devient manifeste en sa propre talité » $(R N, 90)$. La conversion nishitanienne, ne rejoignant aucun Dieu au sens chrétien, exige par conséquent d'être pensée, non comme conversion au néant tel que communément entendu — et dont la quaestio a révélé le caractère intenable -, mais comme conversion au phénomène lui-même. Alors que les Confessions peuvent être lues comme l'itinéraire qui conduit Augustin vers sa rencontre de Dieu, Qu'est-ce que la religion? dispose le plan donnant seul lieu à une véritable rencontre avec le manifeste:

La vacuité est le champ sur lequel peut avoir lieu une rencontre essentielle entre des entités normalement tenues pour être les plus distantes l'une de l'autre $[. .$.$] Cette rencontre est dite «essentielle» parce qu'elle prend place$ à la source de l'existence commune à la fois à l'un et à l'autre, et cependant en un point où chacun est véritablement soi-même. $(R N, 102)$

Mais, soucieux de rigueur, le philosophe nuance immédiatement son propos. Si toute rencontre suppose au moins deux termes, dès lors que l'on se situe sur le plan de la vacuité «il est même inadéquat de parler encore d'une "rencontre" » $(R N, 102)$. À ce terme, il vaudra mieux préférer celui de "réalisation », qui avait été proposé dès les premières pages de l'ouvrage. "Réaliser» est en effet pris au double sens qu'il a en anglais aussi bien qu'en français d'éveil à et d'accomplissement. Pour Augustin comme

32. Voir, par exemple, pour le premier sens, Civ. Dei XXII, 29, 1: «Par face de Dieu (facies Dei) nous devons comprendre sa manifestation (manifestatio eius). » Pour le second, In Ep. Ioann. ad Parth. tr. 1, 1: «Ergo manifestata est ipsa vita in carne, quia in manifestatione posita est». Sur tout ceci, voir Giraud (2013). 
pour Nishitani, la conversion est un éveil à la réalité qui s'accompagne d'un devenir réel du moi. Mais chez le penseur de Kyōto, en l'absence de tout Dieu-Forme, c'est dans l'acte de réalisation ainsi entendu qu'il faut chercher le seul terme consistant que la réalité puisse offrir. La réalité se fait en même temps que nous nous éveillons à elle:

J'utilise le mot [«réaliser»] pour exprimer que notre capacité à percevoir la réalité signifie que la réalité se réalise (s'actualise) elle-même en nous; que ceci, à son tour, est la seule manière dont nous pouvons réaliser (nous approprier par la compréhension) le fait que la réalité est ainsi en train de se réaliser en nous; et que lors de ce processus a lieu l'auto-réalisation de la réalité elle-même. ( $R N, 5$, Les parenthèses sont de Nishitani)

La conversion nishitanienne ne se retourne donc pas vers un principe alternatif au moi et à sa finitude, mais elle ne rompt avec la forme de la conscience oppositionnelle que pour renouer avec l'élément même de l'apparaître. Cet élément, elle le retrouve parce que, se découvrant une avec lui, elle le recueille et ainsi l'accomplit comme phénomène.

\section{Conclusion}

Nous avons dans ces pages tenté de dérouler la trame augustinienne, au fil d'une lecture de Qu'est-ce que la religion?. Le schème quaestio-conversioconfessio a révélé son caractère structurant pour toute l'entreprise de Nishitani, dont il permet de revisiter les principales thèses. L'augustinisme s'y trouve en effet mis au service d'une quête philosophique qui se conçoit elle-même comme conquête d'un "point de vue » adéquat sur le réel ${ }^{33}$. Or, l'originalité propre d'Augustin à cet égard est d'avoir prescrit au discours philosophique le point de vue de la confession, non toutefois comme un impératif qui s'imposerait de l'extérieur à la pensée, mais comme la condition requise en vue de son plein exercice. Le point de vue de la confession augustinienne travaille ainsi en profondeur celui de la vacuité nishitanienne. La tâche contemporaine d'un dépassement du nihilisme, issue d'une lecture de Nietzsche et de Heidegger, trouve en Augustin un interlocuteur et un allié, lui dont tout le questionnement émane de l'abîme ouvert par la quaestio. Une dernière interrogation, néanmoins, demeure: si Nishitani, contrairement à Augustin, ne prétend pas rejoindre un quelconque «dieu», pourquoi alors placer toute sa méditation sous le signe

33. Qui est atteint au chapitre IV de l'ouvrage: «The Standpoint of Sūnyatā», (RN, 119167). 
d'une enquête portant sur l'essence de la religion? C'est que le concept de religion ne vaut plus exclusivement ni même d'abord comme relation à un dieu ni comme recherche du salut. Ou plutôt, la place de ce dieu et la chance de ce salut s'identifient désormais avec la réalité comme Proche absolu, dont la rencontre exige que soient outrepassées et abolies les bornes de l'existence subjective. C'est lors de ce décentrement radical hors de l'existence et de ses ressources que se trouve atteint le terrain propre de l'expérience religieuse, et il s'agit finalement d' "approcher la religion [...] comme véritable autoéveil de la réalité ${ }^{34} »(R N, 5)$. Cet autoéveil, aussi nommé «réalisation", constitue le plan propre de la vacuité. Dans le śūnyatā, Nishitani propose ainsi à notre vie et à notre méditation une identification absolue du divin et du phénomène.

\section{Références}

Arendt, H. (1999), Le concept d'amour chez saint Augustin / trad. par A.-S. Astrup, Paris, Payot \& Rivages.

Augustin (1998), Confessions / trad. par E. Tréhorel et G. Bouissou, Paris, Institut des Études Augustiniennes (Bibliothèque augustinienne 13 et $14)$.

Bouton-Touboulic, A.-I. (1999), «De la mort de l'ami à la présence divine (Conf. IV, 4, 7-12, 19) », Vita Latina, 153, p. 58-69.

Bragt, J. van (1989), "Translating Shūkyō to wa nani ka into Religion and Nothingness", dans T. UnNo, dir., The Religious Philosophy of Nishitani Keiji, Berkeley, Nanzan Institute for Religion and Culture/ Asian Humanities Press, p. 5-12.

Chrétien, J.-L. (2002), Saint Augustin et les actes de parole, Paris, PUF.

Dallmayr, F. (1992), "Nothingness and Śūnyatā: A Comparison of Heidegger and Nishitani ", Philosophy East and West, 42/1, p. 37-48.

Elberfeld, R. (2011), "The Middle Voice of Emptiness: Nishida and Nishitani ", dans B. W. Davis et al., Japanese and Continental Philosophy, Bloomington, Indiana University Press, p. 269-285.

34. Voir aussi les deux formules de la page suivante par lesquelles Nishitani expose son projet: «interpréter la quête religieuse comme la recherche par l'homme en direction de la vraie réalité d'une manière qui soit elle-même réelle»; "avancer une réponse à la question portant sur l'essence de la religion en retraçant le procès d'une poursuite réelle de la véritable réalité»(p. 6). 
Giraud, V. (2013), Augustin, les signes et la manifestation, Paris, PUF (Epiméthée).

Greisch, J. (2002), Le Buisson ardent et les lumières de la raison, t. 1, Paris, Cerf.

Heidegger, M. (1996 [1968]), «Die onto-theologische Verfassung der Metaphysik» in Identität und Differenz, GA t. 11, p. 51; trad. fr. par A. Préau, "Identité et différence ", dans Questions I, Paris, Gallimard.

(1995), "Augustinus und der Neuplatonismus", dans Phänomenologie des religiösen Lebens, GA 60 (cours du semestre d'été 1921, Fribourg), Frankfurt, Klostermann, p. 157-299.

Jones, D. (2011), «Empty Soul, Empty World: Nietzsche and Nishitani», dans B. W. Davis et al., Japanese and Continental Philosophy, Bloomington, Indiana University Press, p. 102-119.

Lacoste, J.-Y. (1998), dir., Dictionnaire critique de théologie, Paris, PUF, p. 277-278.

Marion, J.-L. (2008), Au lieu de soi. L'approche de saint Augustin, Paris, PUF.

Matsumaru, H. (1997), «Nishitani's Religionsphilosophie. Nihilism and the Standpoint of śünyatā", Zen Buddhism Today, 14, p. 97-113.

Nishitani, K. (1982), Religion and Nothingness [= Qu'est-ce que la religion?], trad. angl. par J. Van Bragt, Berkeley, University of California Press.

Stambaugh, J. (1995), «Transcendance », The Eastern Buddhist, Printemps, p. $17-28$.

Stevens, B. (2003), "L'ontologie religieuse", Le néant évidé. Ontologie et politique chez Nishitani Keiji, Louvain, Peeters, p. 121-134.

Swanson, P. (1996), "Absolute Nothingness and Emptiness in Nishitani Keiji », The Eastern Buddhist, 29/1, p. 99-108.

Unno, T. (1989), dir., The Religious Philosophy of Nishitani Keiji, Berkeley, Nanzan Institute for Religion and Culture / Asian Humanities Press. 


\section{Résumé}

Nishitani Keiji (1900-1990) a mis en œuvre une reprise féconde des questions propres à la tradition métaphysique occidentale en prenant appui sur la conception bouddhiste de la vacuité (japonais: $k \bar{u}$; sanscrit: śūnyatā). Marchant sur les traces d'Augustin et de sa pensée de la conversion, il a lié aussi étroitement que possible pensée philosophique et quête religieuse. Le nouvel édifice conceptuel élaboré par Nishitani autour de la notion de śūnyatā ne prend en effet tout son sens que pour qui se rend capable d'une conversion au sens religieux du terme, décentrement radical de sa propre existence en direction de la vacuité, et permettant seul un accès véritable à l'être. La saisie par Nishitani de l'essence de la religion le conduit ainsi dans les parages de l'augustinisme, éclairant d'un jour nouveau le possible usage contemporain des catégories bouddhistes.

\section{Abstract}

Nishitani Keiji (1900-1990) proposed a profound rethinking of metaphysical themes beginning from the Buddhist conception of emptiness or nothingness (Japanese: kū ; Sanskrit: śūnyatā). Like Augustine (354-430), the supreme thinker of conversion in the West, he brought philosophical thought and religious quest into the closest possible conjunction. Indeed, the new conceptual apparatus built up by Nishitani around śūnyatā makes sense only for one able to undergo a religious conversion or turning-about, a radical decentering of one's existence in the direction of emptiness, which yields an authentic access to being. His philosophical grasp of the essence of religion brings him into mutually illuminating proximity to Augustine, thus shedding a new light on the possible use of Buddhist categories themselves for contemporary thought. 